\title{
Improving lifetime of quasi-CW laser diode arrays for pumping 2- micron solid state lasers
}

\author{
Farzin Amzajerdian ${ }^{*}$, Byron L. Meadows ${ }^{\mathrm{a}}$, Nathaniel R. Baker ${ }^{\mathrm{b}}$, Bruce W. Barnes ${ }^{\mathrm{a}}$, Upendra N. \\ Singh $^{\mathrm{a}}$, Michael J. Kavaya ${ }^{\mathrm{a}}$ \\ ${ }^{a}$ NASA Langley Research Center, MS 468, Hampton, Virginia 23681-2199 \\ ${ }^{\mathrm{b}}$ Lockheed Martin Engineering and Science Company, Langley Research Center, MS 468, Hampton, \\ Virginia, 23681
}

\begin{abstract}
Operating high power laser diode arrays in long pulse regime of about $1 \mathrm{msec}$, which is required for pumping 2-micron thulium and holmium-based lasers, greatly limits their useful lifetime. This paper describes performance of laser diode arrays operating in long pulse mode and presents experimental data on the active region temperature and pulse-to-pulse thermal cycling that are the primary cause of their premature failure and rapid degradation. This paper will then offer a viable approach for determining the optimum design and operational parameters leading to the maximum attainable lifetime.
\end{abstract}

Keywords: laser diode array, laser diode pump, solid-state laser, lifetime, reliability, lidar, space-based laser instruments.

\section{INTRODUCTION}

Most moderate to high power solid state lasers require high-power quasi-CW laser diode arrays (LDAs) as their optical pump source. Compared with their low-power CW counterparts, these LDAs suffer from shorter lifetimes and are more susceptible to degradation and premature failure. This is mainly due to the excessive localized heating and substantial pulse-to-pulse thermal cycling of the laser active regions. The thermally-induced stresses are even more dramatic when the required pump pulsewidth is increased from 200 microseconds, applied to neodymium-based lasers, to about 1 millisecond required for thulium and holmium lasers ${ }^{1-3}$.

Presently, lifetime testing of a number of LDAs from different suppliers is underway to assess the impact of long pulsewidth operation on the laser lifetime and reliability. The measurements to date indicate a lifetime of more than an order of magnitude shorter than reported lifetime for similar type of arrays operated at 200 microseconds pulsewidth. In addition to shorter lifetime, the arrays experience a high rate of catastrophic failure when operated in long pulse regime. A number of steps can be taken to improve the lifetime of LDAs such as operation at a de-rated level and a reasonably low temperature. Although these considerations will increase the lifetime and reliability of LDAs, an accurate trade analysis between LDA operational and design parameters is required in order to improve lifetime to an acceptably quantifiable level for lidar space missions using thulium/holmium lasers. This paper discusses the experimental methodology and the resulting data revealing the thermal characteristics of different LDAs and provides the basis for a meaningful trade analysis leading to maximum attainable lifetime and reliability. Such a trade analysis can enable optimization of LDA specifications such as package type, number of bars per package and their pitch, operational temperature, and operating parameters such repetition rate, pulsewidth, and applied current

\section{PERFORMANCE IN LONG PULSE MODE}

To asses the impact of operation in the long pulse mode, a number of LDAs of different package types from different suppliers were subjected to a series of measurements including basic power and efficiency versus applied current,

*f.azmajerdian@nasa.gov, phone: 1-757-864-1533, fax: 1-757-864-8828. 
spectral characteristics, thermal properties, and package heat removal efficiency. A subset of these LDAs were then selected for initial lifetime testing in order to evaluate different commercially available LDAs, determine their lifetime expectancy, and to establish a baseline for improving their lifetime and reliability. Because of the lack of any dependable models, these initial lifetime tests were not accelerated and the LDAs were operated at the rated operational current and the expected pulse repetition rate for a space-based 2-micron lidar. The lifetime testing of the first set of LDAs began over two years ago (February 2004) and is now nearing its completion. The lifetime test facility developed for this work was originally capable of simultaneous measurement of 8 LDAs and then expanded to 12 LDAs and recently to 16 LDAs (Fig. 1).

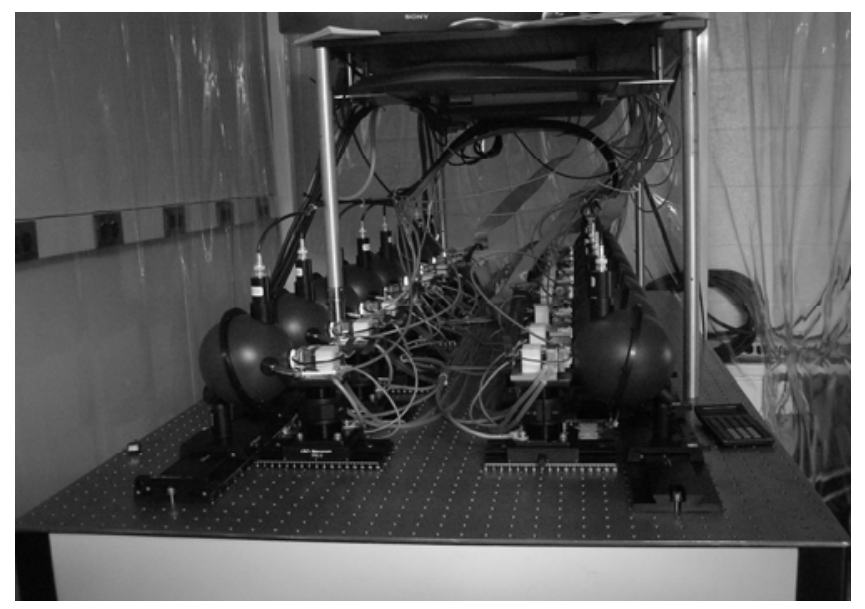

Figure 1. Laser diode array lifetime test facility.

The lifetime test facility is fully automated using a single computer to set operational and environmental parameters, acquire and archive data, flag anomalous readings, and generate a number of warning and status alert messages when necessary. All the LDA performance parameters are continuously monitored and recorded using a common set of instruments for consistency and accurate comparative analysis and evaluation.

In order to provide a basis for comparative investigation of different LDAs, all the measured devices are 6-bar arrays with each bar rated at $100 \mathrm{~W}$ for a total of $600 \mathrm{~W}$ of peak power. All the arrays are tested under the general operational parameters chosen based on a notional earth-orbiting coherent Doppler lidar design listed below:

$\begin{array}{ll}\text { - Drive current } & 70-100 \mathrm{~A} \\ \text { - Pulse duration } & 1 \mathrm{msec} \\ \text { - Rep. rate } & 10-20 \mathrm{~Hz} \\ \text { - Heatsink temp. } & 25 \mathrm{deg} . \mathrm{C}\end{array}$

Fig. 2 is a representative of the lifetime test data showing the peak power versus number of shots for standard "A" and "G" package arrays from one of the major suppliers. As can be seen from Fig. 2, the arrays demonstrate a continuous gradual degradation with the number of shots for both package types. The degradation rate is somewhat lower for the Gpackage arrays but for both package types the lifetime is substantially shorter compared with reported data for operation at shorter pulsewidths $(<200 \mu \mathrm{sec})$. The anticipated lifetime is often represented through a statistical Weibull chart showing the probability of an array's power dropping below a specified level. Fig. 3 shows a Weibull chart of the Fig. 2 data defining a $9 \%$ drop in output power as end of the life and a shape parameter $(\beta)$ of 2.9. Although the number of data points in Fig. 3 is too small for a statically accurate representation of this particular array, it still provides useful information about its lifetime expectancy. Fig. 3 indicates that there is a 1\% probability that this array will fail before it accumulates 30 million shots, $50 \%$ probability of reaching $120 \mathrm{M}$ shots, and that $99.9 \%$ probability of failing by 220 million shots. This lifetime is inadequate for an Earth orbiting 2-micron lidar instrument which will require a lifetime of at least 1.0 billion shots. 


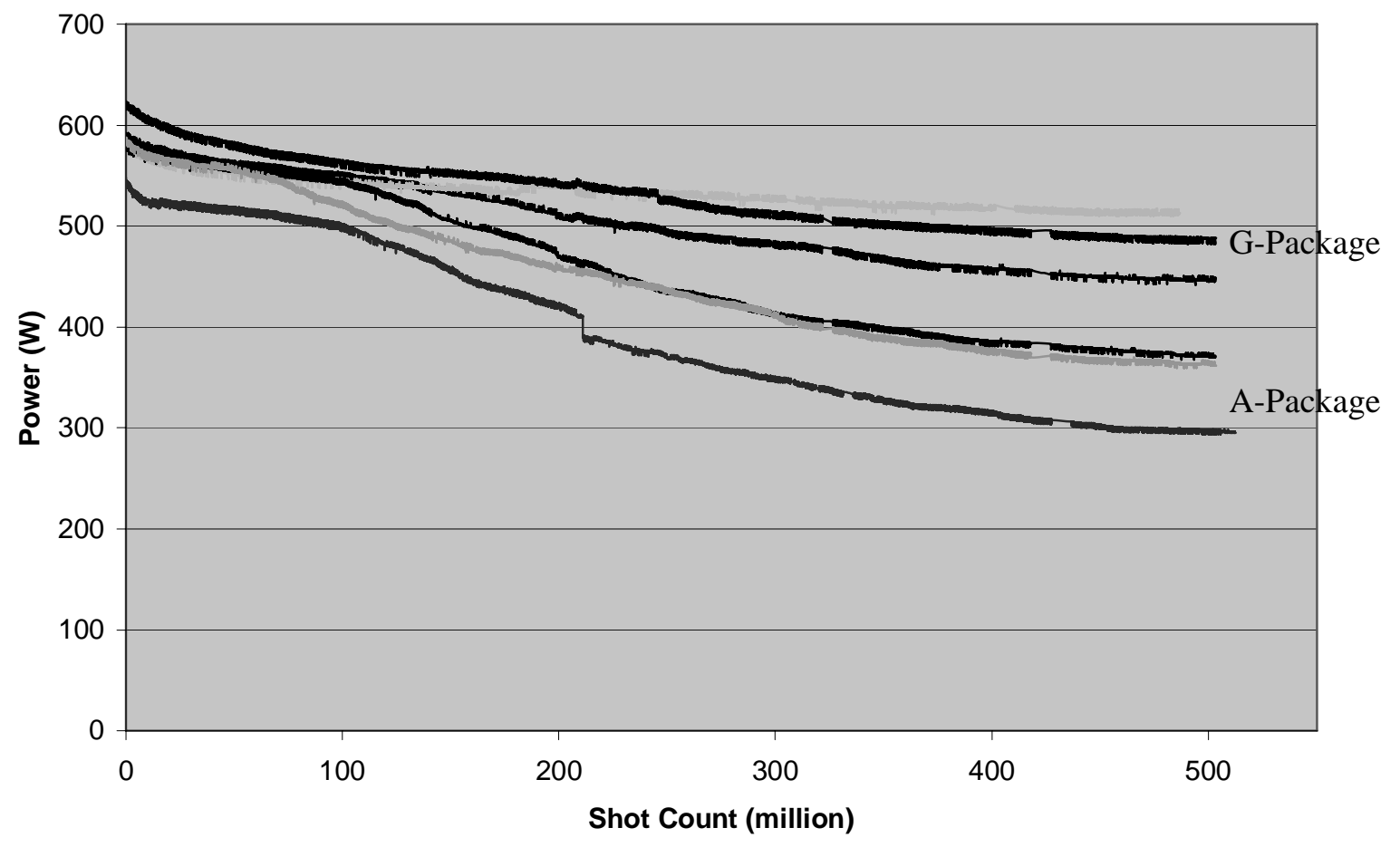

Figure 2. Example of lifetime test data of LDAs operating in long pulse mode.

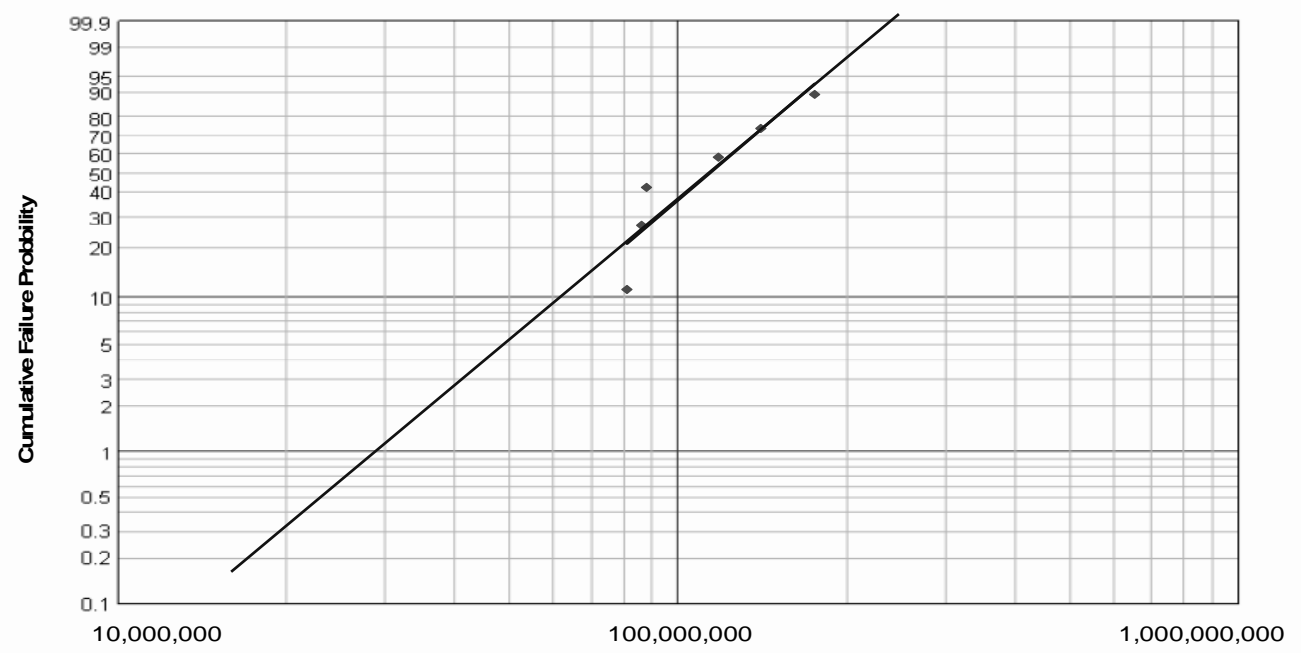

Figure 3. Weibull chart generated from the lifetime data illustrates the expected lifetime.

As noted earlier, the excessive heating and drastic thermal cycling resulting from operation over the long pulse duration of $1 \mathrm{msec}$ are the cause of premature failure and rapid degradation of these LDAs ${ }^{4-8}$. Fig. 4 provides the measured junction temperature of a LDA as a function of pulsewidth showing an increase of about 10 degrees for $1 \mathrm{msec}$ 
pulsewidth operation compared with $200 \mu$ sec. Fig. 5 illustrates the thermal cycling of a typical LDA where the temperature of the LDA face is measured by an infrared thermal camera.

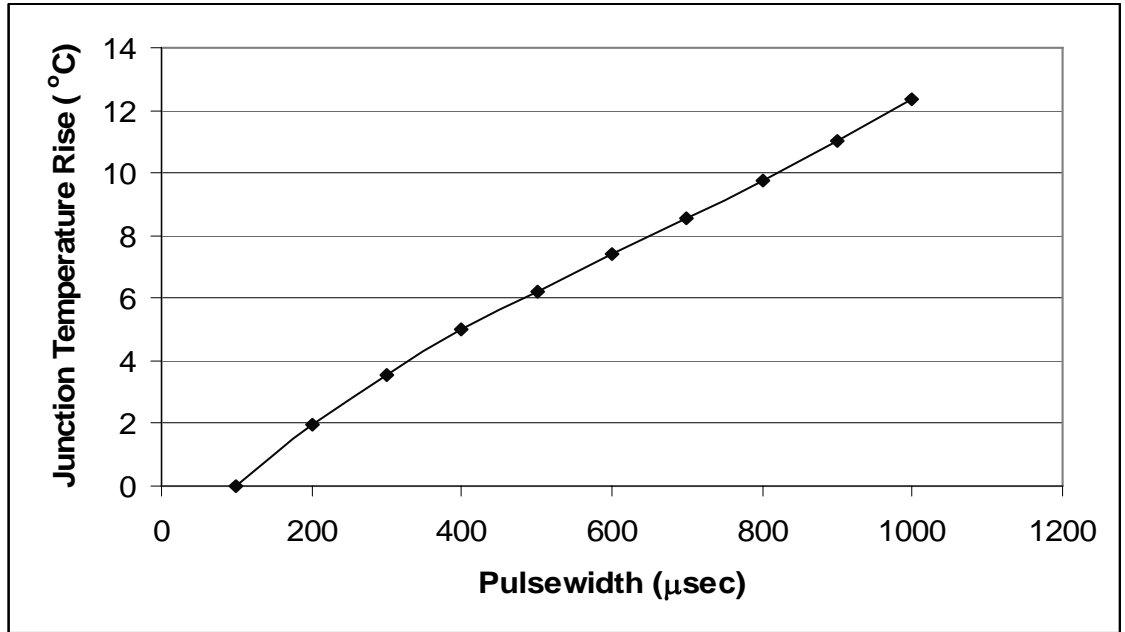

Figure 4. Junction temperature rise versus pulsewidth for a 6-bar laser diode array operating at 70 A.

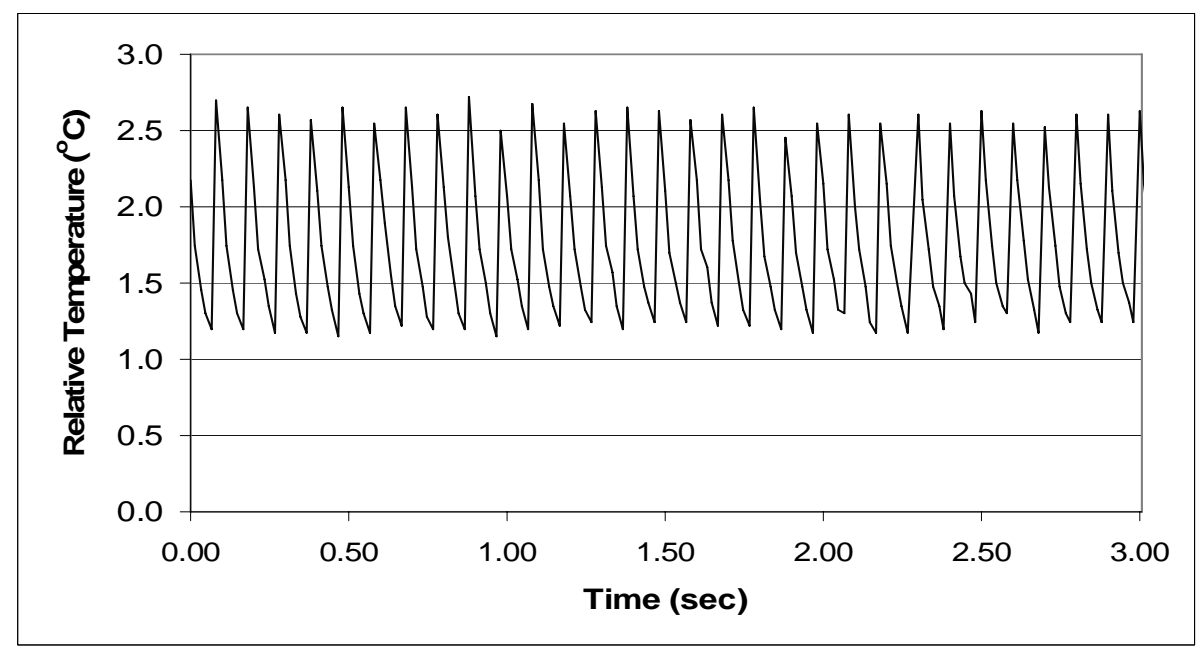

Figure 5. Thermal cycling of an LDA generating 1 msec pulses at $10 \mathrm{~Hz}$

The current LDAs have an electrical to optical efficiency of about 50\%. Therefore, when running a 6-bar LDA close to full rating, about $600 \mathrm{~W}$ of peak power is generated in the form of heat, $(7.2 \mathrm{~W}$ average at $1 \mathrm{msec}$ pulse duration and 12 $\mathrm{Hz}$ prf). This excess energy primarily generated in the active area of the bars (light emitting surface), is quite substantial $^{3-5}$. For a typical bar with approximately $80 \%$ fill factor and $1 \mathrm{~mm}$ cavity length, the average heat flux is about $15 \mathrm{~W} / \mathrm{cm}^{2}$ and peak heat flux is over $1 \mathrm{~kW} / \mathrm{cm}^{2}$. It is this extreme excess heat and the rate and efficiency with which it is removed that drastically affects the laser diode performance, reliability and lifetime. The level of impact of the long pulse operation may be roughly estimated by an Arrhenius relationship written as:

$$
\text { Lifetime }(\tau) \propto\left(\mathrm{T}_{\mathrm{a}}-\mathrm{T}_{\mathrm{b}}\right)^{-\mathrm{N}} \operatorname{Exp}\left(\mathrm{E}_{\mathrm{a}} / \mathrm{kT}_{\mathrm{a}}\right)
$$

Where lifetime $(\tau)$ is expressed as a function of junction temperatures $T_{a}$ and $T_{b}$ measured immediately after and before the generated pulse, the activation energy $\left(E_{a}\right)$ and Boltzmann's constant $(k)$. The leading term accounts for the thermal cycling fatigue due to mismatch of thermal expansion coefficients of different package materials and various layers of the laser bar. The power $\mathrm{N}$ in the expression above can have a value between 2 and 5 depending on the materials 
properties based on the Manson-Coffin law for thermal fatigue. It is obvious from this Arrhenius equation that reducing the temperature difference before and after the pulse is the key for increasing the lifetime to an acceptable level. This may be achieved through careful selection of the LDA package type, specifications of the array considering the pumping requirements, and defining its operational parameters. Table 1 below summarizes the trade space and design and operational specifications that need to be carefully considered based on the solid state laser pump requirements and the lidar mission objectives.

TABLE 1. TRADE SPACE FOR INCREASING THE LIFETIME AND IMPROVING THE RELIABILITY OF LDAS OPERATING IN LONG PULSE MODE.

\begin{tabular}{|c|c|c|}
\hline Trade Space & Specifications & Parameters/Selections \\
\hline \multirow{3}{*}{ Package } & Geometry & A or G package style \\
\hline & Architecture & $\begin{array}{l}\text { Bars in Groove, Rack \& Stack, } \\
\text { Stacked Subassemblies }{ }^{9}\end{array}$ \\
\hline & Heatsink Materials & $\mathrm{BeO}, \mathrm{Cu}, \mathrm{CuW}^{9}$ \\
\hline \multirow{2}{*}{ Bar } & Efficiency & Wafer architecture and Epitaxy \\
\hline & Fill factor & No. of emitters per bar \\
\hline \multirow{2}{*}{ Array } & No. of Bars & $\begin{array}{l}\text { Constrained by pump power } \\
\text { requirements }\end{array}$ \\
\hline & Pitch & $\begin{array}{l}\text { Constrained by pump brightness } \\
\text { requirements }\end{array}$ \\
\hline \multirow{4}{*}{$\begin{array}{l}\text { Operational } \\
\text { Parameters }\end{array}$} & $\begin{array}{l}\text { Drive Current } \\
\text { (de-rating) }\end{array}$ & $\begin{array}{l}\text { Constrained by pump power } \\
\text { requirements }\end{array}$ \\
\hline & Pulsewidth & $\begin{array}{l}\text { Constrained by pump power and } \\
\text { system efficiency requirements }\end{array}$ \\
\hline & $\begin{array}{l}\text { Pulse Repetition } \\
\text { Rate }\end{array}$ & $\begin{array}{l}\text { Constrained by science product } \\
\text { requirements }\end{array}$ \\
\hline & Sink Temperature & $\begin{array}{l}\text { Constrained by spacecraft heat } \\
\text { management system }\end{array}$ \\
\hline
\end{tabular}

\section{THERMAL MEASUREMENTS}

Several experimental tests have been developed to investigate the thermal characteristics of high power LDAs in order to evaluate various package designs and define the best operating parameters. These measurements also facilitate the technology advancement activities leading to the development of new LDAs with improved reliability and lifetime. The thermal characterization of LDAs includes the thermal imaging of the facets, spectral shift and broadening measurements, and a forward voltage measurement technique we refer to as the "Forward Voltage-Short Pulse" (FV-SP) technique. The FV-SP technique is devised based on a conventional measurement used for electrical diodes, lightemitting diodes, and continuous-wave laser diodes. The FV-SP measurements are particularly useful in providing the necessary data for a meaningful trade analysis leading to maximum attainable lifetime and reliability. Such a trade analysis can encompass all the LDA design and operational parameters as summarized in Table 1 in addition to facilitating quantitative comparison between vendors and packages and screening of devices within a given fabrication run.

The FV-SP measurement utilizes the diode characteristics of the LDA to measure its junction temperature. In this measurements, a series of relatively short and low current pulses, compared with the actual drive current, are applied to the LDA and the resultant voltage are measured with a high degree of precision (Fig. 6). The measured voltage drop across the array is related to the junction temperature through the diode I-V equation. 


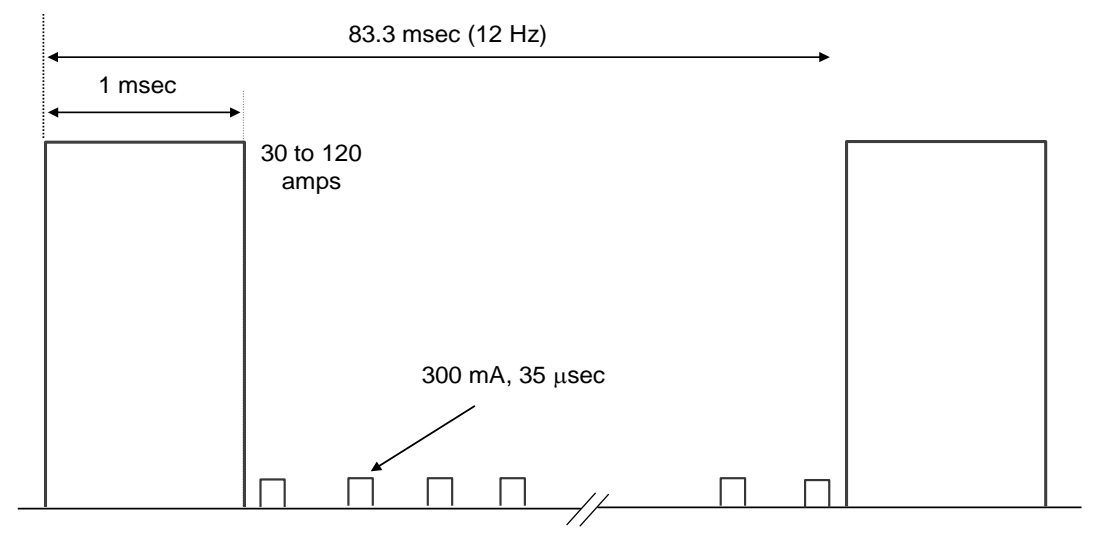

Figure 6. The Forward Voltage-Short Pulse technique for measuring the LDA junction temperature.

One of the main advantages of this technique is its ability to obtain the junction temperature before and after each LDA pulse. An additional benefit of this measurement technique is its ability to determine the junction temperature while running the LDA at any operational parameters without tedious post processing required by other techniques such as time-resolved spectral measurements. Fig. 7 is an example of the FV-SP measurements showing the peak junction temperature before and after the generated pulses and the thermal impedance (ratio of junction temp. rise to dissipated heat) as a function of drive current. It can be seen from these plots that the temperature rise during the pulse is almost a linear function of applied current. Using the measured junction temperatures in the Arrhenius expression of previous section, the relative impact of current de-rating can be estimated. Fig. 8 illustrates the lifetime improvement resulting from reducing applied current. It worth noting that high power quasi-CW laser diodes arrays are complex electro-optical components and thus their lifetimes do not follow well defined or known predictable models such as Arrhenius relationships unless considerable statistical data is available for accurately specifying the activation energy (Ea) and thermal fatigue constant $(\mathrm{N})^{10-13}$. However, results such as shown in Fig. 8 can still provide useful information by enabling a determination of the magnitude of improvement that can result from various measures for reducing the junction temperature and thermal cycling. Fig. 8 clearly shows that up to an order of magnitude improvement in lifetime may result from de-rating by about 30\% (i.e., operating at $70 \mathrm{~A}$ ). Fig. 9 is another example of the FV-SP measurements showing the thermal impedance of a LDA versus pulsewidth while maintaining a fixed duty factor (pulsewidth X rep. rate $=$ Const.). Relative expected lifetime is also shown in this figure and indicates that a two order of magnitude longer lifetime is possible when operating at $200 \mu \mathrm{sec}$ and shorter pulses compared with 1 msec operation.

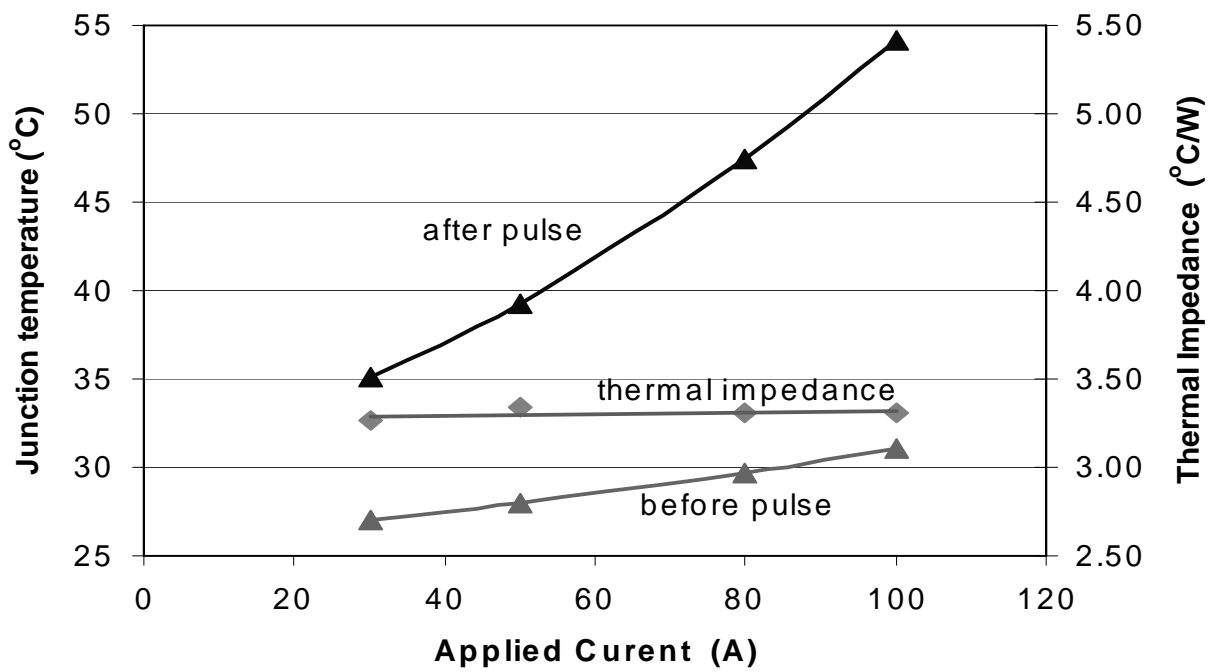

Figure 7. Before and after pulse junction temperatures measured using the Forward Voltage-Short Pulse (FV-SP) technique. 


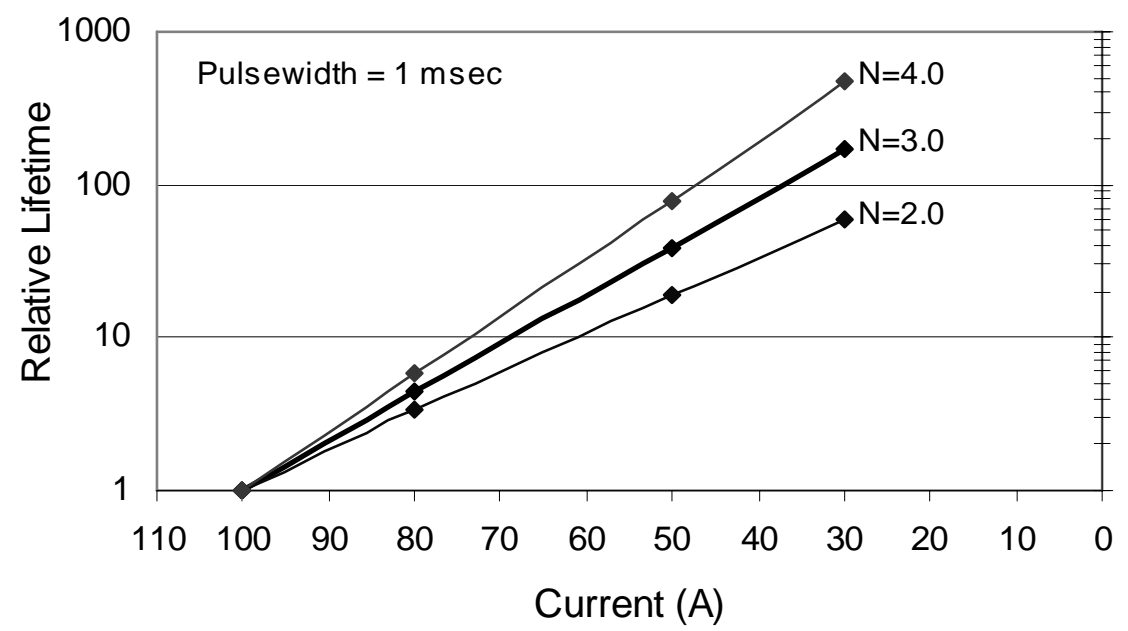

Figure 8. Relative lifetime expectancy versus applied current for different values of thermal fatigue constants.

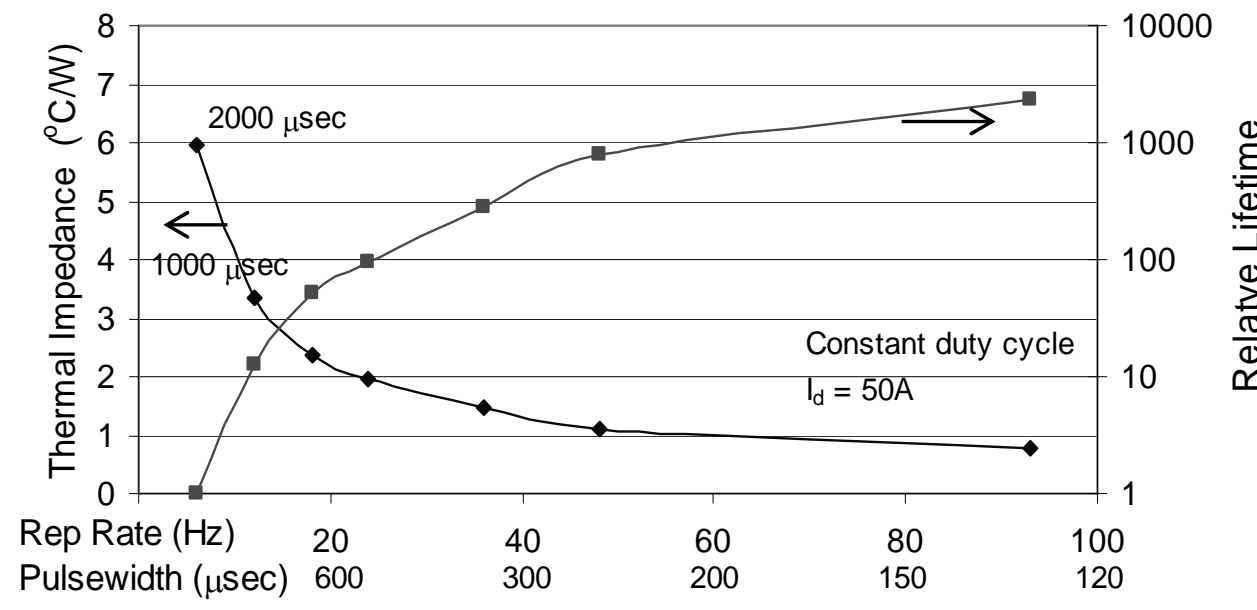

Figure 9. Thermal impedance and relative lifetime as a function of pulse width and repetition rate while keeping the total average power constant.

As Figs. 7-9 illustrate, using the thermal data obtained from FV-SP measurements in an Arrhenius model allows for a reasonable analysis of the LDA performance and determining its optimum operational parameters for achieving the maximum possible lifetime while meeting the lidar and the mission requirements

\section{CONCLUSION}

Measurements to date indicate that operating off-the-shelf LDAs over long pulse duration regime of the order of $1 \mathrm{msec}$ substantially degrade their performance. The LDA lifetime can be increased by careful specification of the LDA and by determining its optimum operational parameters for maximum lifetime by considering the solid state laser pump requirements and the mission objectives. There are also several promising LDA packaging technologies that can address the thermal issues of high power arrays leading to more reliable and longer lifetime. Improvement of LDA lifetime and 
reliability require accurate characterization of their critical thermal properties, primarily the junction temperature of their bars and thermal impedance of their package. Therefore, a series of measurement techniques for quantifying the thermal properties of the LDAs were employed. These measurement techniques include spectral shift and broadening measurements, time-resolved spectral measurement, and high spatial resolution thermal imaging of the LDA facet. In addition to these measurements, a new technique was devised, that we refer to as "Forward Voltage-Short Pulse" (FVSP) technique. Preliminary results of the FV-SP have already provided valuable criteria for de-rating of the LDAs and specifying its operational temperature. This measurement also provides useful data for comparison and evaluation of different package types from various suppliers and specifying their design (ex., geometry, number of bars, and pitch).

\section{ACKNOWLEDGMENTS}

The authors would like to express appreciation to NASA's Earth Science Technology Office for funding this effort through the Laser Risk Reduction Program.

\section{REFERENCES}

1. N. P. Barnes, W. J. Rodriguez, and B. M. Walsh, “Ho:Tm:YLF laser amplifiers,” J. Opt. Soc. Am. B, 13, 28722882, 1996.

2. N. P. Barnes, E. D. Filer, F. L. Naranjo, W. J. Rodriguez, and M. R. Kokta, "Spectroscopic and lasing properties of Ho:Tm:LuAG,” Opt. Lett, 18, 708-710, 1993.

3. J. Yu,, A. Braud, and M. Petros, “600mJ, Double pulsed 2 micron laser,” Opt. Lett., 28, 540-542, 2003.

4. H. Brugger and P. W. Epperlein, "Mapping of local temperatures on mirrors of GaAs/AlGaAs laser diodes," Appl. Phys. Lett., 56, 1049-1051, 1990.

5. Anna Kozlowska, et al, "Thermal properties of high-power lasers investigated by micro-thermography," SPIE Proc, 2711, 2005.

6. B. Laikhtman, A. Gourevitch, D. Donetsky, D. Westerfeld, and G. Belenky, "Current spread and overheating of high power laser bars,” J. Appl. Phys., 95, 3880-3889,2004.

7. N. I. Katsavets, et al, "Study of correlation between optical characteristics and mirror facet temperatures of the active region in high power SCH SQW InGaAsP/GaAs laser diodes," SPIE Proc, 2148, 152-156, 1994.

8. W. C. Tang, et al, "Raman microprobe study of the time development of AlGaAs single quantum well laser facet temperature on route to catastrophic breakdown,” Appl. Phys. Lett., 58, 557-559, 1991.

9. B. L. Meadows, F. Amzajerdian, B. W. Barnes, N. R. Baker, R. P. Baggott, U. N. Singh, and M. J. Kavaya, "Reliability of Long Pulsewidth High Power Laser Diode Arrays," Proc. of Materials Research Society, Symposium on Advanced Devices and Materials for Laser Remote Sensing, V. 883, 2005.

10. H. C. Casey and M. B. Panish, Heterostucture lasers, Academic Press, NY, 1978.

11. M. Fukuda, "Reliability and degradation of semiconductor lasers and LEDs," Artech House Inc., MA, 1991.

12. F. Dorsch and F. Daiminger, "Aging test of high power diode lasers as a basis for an international lifetime standard,” SPIE Proc, 2870, 381-389, 1996.

13. B. Lu, E. Zucker, et al, "High power, high reliability CW and QCW operation of single AlGaAs laser diode array design,” SPIE Proc, 3945, 293-300, 2000. 